\title{
Using break quantities for tactical optimisation in multistage distribution systems
}

\author{
Marcel J. Kleijn Rommert Dekker \\ Econometric Institute \\ Erasmus University Rotterdam \\ P.O. Box 1738, 3000 DR Rotterdam \\ The Netherlands
}

\begin{abstract}
In this chapter we discuss a tactical optimisation problem that arises in a multistage distribution system where customer orders can be delivered from any stockpoint. A simple rule to allocate orders to locations is a break quantity rule, which routes large orders to higher-stage stockpoints and small orders to end-stockpoints. A so-called break quantity determines whether an order is small or large. We present a qualitative discussion on the implications of this rule for the marketing process, and a qualitative and quantitative analysis on the implications for the transportation and inventory costs. Furthermore, we present a case study for a company that implemented a break quantity rule. Finally, in the last section the main results are summarised.
\end{abstract}

Keywords. Distribution systems, inventory, transportation, marketing, break quantity rule

\section{Introduction}

Distribution systems are concerned with the effective management of the delivery of finished goods to the final customers. Since in general there are many complex interactions between the components of such a system, the decision process can be extremely difficult. 
Therefore, in most cases the decisions are decomposed into strategical, tactical and operational decisions. Strategical decisions include the determination of the location and size of factories and warehouses, the design of transportation facilities, and so on. Tactical decisions cover the problem of how to use the resources in such a way that customer demand is met at minimum cost or maximum service. Operational decisions involve all day-to-day operational and scheduling decisions. Since an integral approach of these categories is impossible (see e.g. Tüshaus \& Wahl (1997)), one often uses an hierarchical approach, where first the strategical decisions are made, followed by the tactical and operational decisions.

In this chapter we discuss a tactical problem that occurs when at the strategical level the decision is made that customer orders can be delivered both from end-stage stockpoints (say, warehouses) and from higher-stage stockpoints (e.g. central warehouses or distribution centres) or factories. Deliveries from a higher-stage stockpoint or factory will henceforth be referred to as direct deliveries (Fleischmann $(1993,1997)$ ). Direct deliveries can be advantageous because bypassing a warehouse results in shorter distances and saves warehousing (handling, storage) costs. However, there may also be a loss in transportation economies of scale, thus raising the transportation costs. If direct deliveries are allowed, then upon arrival of a customer order a decision has to be made from which location to deliver this order. In principle, an optimal decision will depend on the locations of the customer, the factories and the warehouses, on the size of the order, on the stock levels at the factories and warehouses, on the amount in transit to the warehouses and on the maximum delivery lead time quoted by the customer. Furthermore, if it is possible to combine the delivery of orders, an optimal decision will also depend on orders by other customers. In practice, there is a need for rules that are easy to understand and implement. A simple way to allocate orders to stockpoints is to route large orders to the nearest higher-stage stockpoint or factory, and small orders to the nearest warehouse (see e.g. Ballou (1992), Fleischmann (1993, 1997)). This rule will be called a break quantity rule, where a so-called break quantity determines whether an order is small or large. The implementation of such a rule in logistics software is very simple. Some standard packages already include the option of setting a maximum issue quantity. The large orders may then be allocated automatically or by the logistics manager.

Applying a break quantity rule will have a number of opposite effects on the costs and service level in a distribution system, thus making the determination of a good break quantity a difficult task. In most distribution networks a weight limit of 1 or 2 tons is used (Fleischmann (1997)). However, this number is usually based on experience and intuition, rather than on a quantitive analysis. In this chapter we will discuss how to determine a 
good break quantity by carefully examining the relevant costs. In the next sections we analyse the implications of a break quantity rule on the performance of a distribution system, and we present a discussion on the determination of the break quantity. In Section 4 a case study is discussed, in which some additional complexities that may arise in practice are addressed. The main results are summarised in the last section.

\section{Implications of a break quantity rule}

In this section the influence of a break quantity rule on the performance of a general distribution system is analysed. In particular, we discuss the effects on the marketing process, the transportation and handling costs, and the inventory costs.

An important motivation for using warehouses in a distribution system is the improved customer service that is caused by shorter delivery lead times. Fleischmann (1993, 1997) observed that the high level of competition in many consumer goods markets has caused the customers to claim a better distribution service, in particular shorter delivery lead times and more frequent deliveries of smaller amounts. Nevertheless, the introduction of a break quantity rule may lead to longer delivery lead times for large orders, for example if small orders are delivered from stock and large orders are handled on a produce-to-order basis. This situation was described in a case study (Nass, Dekker \& Sonderen-Huisman (1997)) for a company that applied a break quantity rule. However, Kok \& Janssen (1996) argue that a major reason for the occurrence of occasional large orders is the discount structure used by companies to increase sales, and the need for immediate delivery is much less for these large orders. Also, a customer placing a large order may be the manager of another warehouse, and the delivery lead time can be negotiated upon. Therefore, we conjecture that the possible increase in delivery lead time has the least negative marketing effects for large orders. Finally, in many practical situations, the arrival of an unexpected large order causes the management to make an ad-hoc decision whether or not to deliver the order from stock. Applying a break quantity rule on the one hand means less flexibility for the management, but on the other hand it creates a consistent view towards customers. Upon order entry, direct feedback can be given about the delivery of the order. If a customer does not accept the break quantity rule and considers placing his large order at a competitor (e.g. because of an increase in delivery lead time for the large order), he may be convinced by offering a price rebate (Kasturi Rangan \& Jaikumar (1991), Kok \& Janssen (1996)), which can be financed by the reduction in transportation, handling and inventory costs. If the customer still can not be convinced, then an exception can be made for him. 
Transportation costs are mainly depending on distance and shipment size. Due to economies of scale, these costs are typically an increasing and concave function of the distance or the shipment size (Ballou (1992)). The influence of a break quantity rule on the transportation costs is very difficult to predict, because there are opposite effects. On the one hand, since direct deliveries are always shorter than deliveries via a warehouse, the transportation costs will decrease. On the other hand, some economies of scale are lost because shipments that used to be consolidated in replenishment orders to the warehouse are now shipped directly. This causes the transportation costs to increase. If customers are located near the warehouse, then it is likely that direct deliveries are more expensive than deliveries via the warehouse. However, this cost increase is minimised if direct deliveries are preserved for large orders. A perfect situation arises if a large order implies a full truck load, since in this case a break quantity rule has no increasing effect on the transportation costs. Finally, we observe that a break quantity rule implies that part of the demand is bypassing the warehouse, and thus the handling costs are reduced.

If a break quantity rule is applied, then at the warehouses the peaks in demand are filtered out, which results in a reduction of the average demand at the warehouses, a reduction of the average stock in transit to the warehouses, and a reduction of the demand variability at the warehouses. Hence, the inventory holding costs at the warehouses will decrease. The greatest reduction is obtained for items having an erratic demand pattern, i.e. items which have occasional very large demand transactions interspersed among a majority of small transactions (Silver (1970)). Safety stock levels for such items tend to be quite large in order to meet certain service requirements. Orders that are not allocated to the warehouses must be delivered from another location. If this location is e.g. a factory that produces to order and does not keep inventory, a break quantity rule will have no inventory effect here. But, if large orders have priority over replenishment orders, a break quantity rule may cause an increase in production costs. If the other location holds inventory, say a central warehouse which supplies regional warehouses, there will be a negative effect on the inventory costs, i.e. they will increase. The central warehouse will now face the occasionally occurring large orders, which increases the demand variability and thus leads to higher inventory costs. However, if large orders from several regional warehouses are allocated to the same central warehouse, the centralisation effect (Eppen (1979)) at this warehouse induces the inventory costs to decrease (see also Dekker, Kleijn \& Kok (1997)). As was the case for the transportation costs, the net effect on the central warehouse inventory costs is difficult to predict.

To conclude this section, we summarise in Table 2.1 the main advantages and disadvantages 
of using a break quantity rule instead of a traditional policy where all demand is delivered from the warehouse.

Table 2.1: Main advantages/disadvantages of break quantity rule

\begin{tabular}{ll}
\hline advantages & disadvantages \\
\hline 1. total transportation distance decreases & 1. longer delivery lead times \\
2. less stock needed at warehouse & 2. less transportation ec. of scale \\
3. less handling & $\begin{array}{l}\text { 3. more stock needed at } \\
\end{array}$ \\
& central warehouse or factory \\
\hline
\end{tabular}

\section{Determining the break quantity}

When a break quantity rule is applied, some important decisions have to be made. For example, a company having customers from different regions can set a different break quantity for each region, or one break quantity for all regions. Although the first option slightly decreases the consistency towards the customers, it allows for a better trade-off between inventory and transportation costs, since transportation costs are in most cases region dependent. Another important issue is the determination of the break quantity. Such a decision will usually be made using a qualitative analysis with respect to the marketing process and a quantitative analysis with respect to the transportation and inventory costs. As far as the marketing process is concerned, we assume that the break quantity rule is accepted by the customers and thus will have no effect on the total demand. In the next subsections the quantitative analysis with respect to the transportation and inventory costs is discussed, for a simple distribution system consisting of one factory, one warehouse and one customer region.

\subsection{Notation and assumptions}

Consider a simple distribution system consisting of a factory, a warehouse, and some customers located in the same region. It is assumed that a break quantity rule is applied, with a break quantity equal to $q$. The system is illustrated by Figure 3.1.

It is also assumed that the demand process can be described by a compound Poisson process, with arrival rate $\lambda$. Upon arrival, a customer places an order for $j$ units with probability $a(j), j=1, \ldots, \infty$. The main reason for modelling demand in this way is because it allows us to distinguish between customers based on their order sizes. The distribution of the demand during a period of $t$ time units, given a break quantity $q$, has 
Figure 3.1: The distribution system

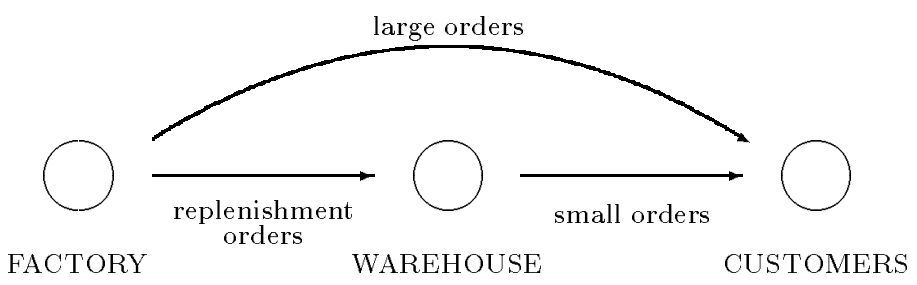

a density function denoted by $f_{q}^{t}$. Using Adelson's recursion scheme (Adelson (1966)), it can be shown that this density function satisfies the following recursive relations

$$
f_{q}^{t}(j)= \begin{cases}e^{-\lambda t\left(1-a_{q}(0)\right)} & \text { if } j=0 \\ (\lambda t / j) \sum_{i=0}^{j-1}(j-i) a_{q}(j-i) f_{q}(i) & \text { if } j=1,2, \ldots\end{cases}
$$

where

$$
a_{q}(j):= \begin{cases}\sum_{i=q+1}^{\infty} a(i) & \text { if } j=0 \\ a(j) & \text { if } 1 \leq j \leq q \\ 0 & \text { otherwise }\end{cases}
$$

Large orders are produced to order at the factory and shipped to the customer as soon as possible. Small orders are delivered from stock on hand if possible, and demand which can not be satisfied directly from stock on hand is backlogged. The scheduling of the replenishment orders is assumed to be determined by the inventory policy.

\subsection{Transportation costs}

In many situations, the transportation of finished goods to customers is contracted out (Ballou (1992), Fleischmann (1993)). In this case we define the following relevant transportation tariffs:

$T_{f w}(i)$ : the costs of shipping $i$ units from the factory to the warehouse

$T_{w c}(i)$ : the costs of shipping $i$ units from the warehouse to a customer

$T_{f c}(i)$ : the costs of shipping $i$ units from the factory to a customer

A typical transportation tariff consists of a minimum charge, and (decreasing) transport rates for several weight classes (Ballou (1992), Fleischmann (1993)). 
For a given break quantity $q$, the expected transportation costs per time unit for shipments to the customers are given by

$$
\lambda \sum_{j=1}^{q} T_{w c}(j) a(j)+\lambda \sum_{j=q+1}^{\infty} T_{f c}(j) a(j)
$$

The average cost for the transportation of replenishment orders depends on the inventory policy. For example, if a replenishment order is placed every $R$ time units, the expected transportation costs per time unit are given by

$$
(1 / R) \sum_{j=1}^{\infty} T_{f w}(j) f_{q}^{R}(j)
$$

If replenishment orders are always shipped in batch sizes equal to $Q$, these expected costs become

$$
(1 / Q) T_{f w}(Q) \sum_{j=1}^{\infty} j f_{q}(j)=(1 / Q) T_{f w}(Q) \lambda \sum_{j=1}^{q} j a(j)
$$

Observe that $\sum_{j=1}^{\infty} j f_{q}(j)=\lambda \sum_{j=1}^{q} j a(j)$ denotes the average demand for the warehouse per time unit, if the break quantity equals $q$. Many times in practice replenishment orders are transported in full truck loads, implying that the transportation tariff for these orders is linear in the shipment size (e.g. Nass, Dekker \& Sonderen-Huisman (1997), Fleischmann (1997)). This is possible if for example replenishment orders for different items are combined in one shipment. In this case the expected transportation costs are given by

$$
T C(q)=\lambda \sum_{j=1}^{q} T_{w c}(j) a(j)+\lambda \sum_{j=q+1}^{\infty} T_{f c}(j) a(j)+\lambda \sum_{j=1}^{q} t_{f w} j a(j)
$$

with $t_{f w}$ the transportation rate for replenishments orders. Observe that these costs are independent of the inventory policy at the warehouse, since the scheduling of the replenishment orders no longer affects the transportation costs. It is now relatively easy to analyse the effect of a break quantity rule on the transportation costs.

\subsection{Inventory costs}

A break quantity rule can be combined with any inventory policy at the warehouse, since it only influences the demand distribution. If the inventory policy parameters were first determined based on a compound Poisson distribution with arrival rate $\lambda$ and order size distribution $a(j), j=1, \ldots, \infty$, the new parameters can be set in a similar way for a 
compound Poisson demand distribution with the same arrival rate and order size distribution $a_{q}(j), j=1, \ldots, q$ (see $\left.(1)\right)$. Examples of such approaches are given in Silver (1970), Hollier, Mak \& Lam (1995a, 1995b) and Mak \& Lai (1995a, 1995b).

Most inventory control systems operate with approximative models, which are reasonable since the total cost curve usually has a flat bottom, so that slight deviations from optimum values of the policy parameters result in only small changes to the total costs. Therefore, as an example, we now discuss an approximative inventory model, where the only relevant demand information is contained in the mean and variance of the demand per time unit. For a given break quantity $q$, it can be shown (see e.g. Tijms (1994)) that this mean $\mu_{q}$ and variance $\sigma_{q}^{2}$ are equal to

$$
\begin{aligned}
\mu_{q} & =\lambda \sum_{j=1}^{q} j a(j) \\
\sigma_{q}^{2} & =\lambda \sum_{j=1}^{q} j^{2} a(j)
\end{aligned}
$$

Suppose we have an inventory system where every $R$ time units a replenishment order is placed which arrives $L$ time units later, and management has implied the restriction that the probability of a stockout during the lead time plus review time is less than $\alpha$, with $0<\alpha<1$. With $K$ the fixed cost for placing a replenishment order and $h$ the unit holding cost, we obtain that (see e.g. Ballou (1992)) an approximation for the average costs is given by

$$
I C(q)=(1 / R)\left(K+\frac{1}{2} h \mu_{q} R+h z \sigma_{q} \sqrt{R+L}\right)
$$

with $z:=\Phi^{-1}(\alpha)$ and $\Phi$ the standard normal distribution. If the pipeline inventory is also taken into account, the average costs become

$$
I C(q)=(1 / R)\left(K+h \mu_{q}\left(\frac{1}{2} R+L\right)+h z \sigma_{q} \sqrt{R+L}\right)
$$

One can observe that the inventory costs are increasing with $\mu_{q}$ and $\sigma_{q}$, and thus with the break quantity $q$. For higher service levels, the value of $z$ tends to be higher, and the inventory costs become more sensitive to the standard deviation of the demand. Also, the lead time for replenishment orders has a significant influence on the inventory holding costs.

Optimising the inventory costs with respect to the break quantity always leads to a break quantity of zero, because no inventory is maintained at the factory. However, in this case all orders are shipped directly from the factory, which may lead to very high transportation 
costs. Therefore, the optimisation of the break quantity should be based on both the inventory and the transportation costs. In Dekker et al. (1997) it is shown that in general the average cost function does not have a shape that allows for the design of a straight optimisation algorithm. However, using enumeration only over values of $q$ satisfying $a(q)>$ 0 it is possible to determine the break quantity that minimises the average transportation and inventory costs.

\section{A case study}

Recently, we analysed a company in Western-Europe that applied a break quantity rule. The company produces technical thermoplasts in many different grades and colours. About $50 \%$ of the total volume is produced to order, which corresponds to $90 \%$ of the product varieties. The remaining volume is produced to stock. Customers are located all over the world, but most of them are located in Europe. The company has four production plants, located in different countries (Spain, France, Scotland and the Netherlands), and in each of these plants different products are manufactured. Furthermore, the company has one distribution centre, located in the Netherlands, in which different orders for the same customer are consolidated and shipped to the customer at the end of every week. Orders for produce-to-order products can either be delivered directly to the customer, or they can first be shipped to the distribution centre, where they are consolidated with other orders from that customer. Orders for produce-to-stock products are delivered from stock on hand at the distribution centre, and in case of a stockout the order will be handled as a produceto-order product. If a customer places an order for a product, the company immediately promises a certain delivery date. Reliability of the delivery date is very important for the company, more important than the actual lead time. The break quantity rule is implemented such that an order for a produce-to-order product is shipped directly to the customer if the size of the order exceeds the break quantity, and it is shipped via the distribution centre otherwise. Since the location of the customer has a large impact on the transportation costs, the company asked us to determine a break quantity for each region. The transportation to the customers is contracted out, while the replenishment orders are shipped to the distribution centre with full truck loads. Hence, the costs for replenishment orders are proportional to the shipment size. Also, the handling and inventory costs were assumed to be proportional to the shipment size. The inventory costs for the produce-toorder products were low compared to the transportation costs, since on average the goods were kept in stock at the distribution centre for only three days. 
As in Section 3.2, the transportation tariff for replenishment orders is denoted by $t_{f w}$, where it is assumed that this rate includes the handling and inventory costs at the distribution centre. Moreover, the transportation costs for shipping $j$ units from the production plant to a customer in region $r$, resp. from the distribution centre to a customer in region $r$, are denoted by $T_{f c}^{r}(j)$ and $T_{w c}^{r}(j)$ respectively.

The main problem we encountered was the following: whenever a customer places an order there is no information available on other outstanding orders from the same customers. Hence, it is impossible to determine the transportation costs from the distribution centre to the customer, because the total shipment size is not known. However, it was possible to determine an upperbound on the optimal break quantity, such that direct deliveries of orders with sizes exceeding this upperbound are cheaper than deliveries via the distribution centre. To obtain this upperbound, we observe that the transportation cost rate for shipments from the distribution centre to customers in region $r$ is bounded from below by the full truck load cost rate, i.e. $t_{w c}^{r}:=\lim _{j \rightarrow \infty} T_{w c}^{r}(j) / j$. Hence, the costs of delivering an order of $j$ units via the distribution centre can never be smaller than $\left(t_{f w}+t_{w c}^{r}\right) j$, so an upperbound for the optimal break quantity in region $r$ is given by

$$
\min \left\{j \geq 0: T_{f c}^{r}(i) \leq\left(t_{f w}+t_{w c}^{r}\right) i \text { for all } i \geq j\right\}
$$

As an example we consider a customer region in Germany and the production plant in Spain. The tariffs for transportation from the plant in Spain to the customers in Germany and from the distribution centre in the Netherlands to the customers in Germany are given in Table 4.1.

Table 4.1: Transportation rates (Dfl/100 kg)

\begin{tabular}{l|ccccccc}
\hline & \multicolumn{7}{|c}{ shipment size (tons) } \\
& minimum & $0-0.1$ & $0.1-0.5$ & $0.5-1$ & $1-2$ & $2-3$ & $3-4$ \\
\hline Spain-Germ. & 0 & 89.32 & 75.72 & 69.19 & 62.26 & 57.89 & 48.31 \\
Neth.-Germ. & 0 & 65.00 & 32.50 & 24.20 & 16.60 & 13.00 & 11.20 \\
\hline & $4-5$ & $5-7.5$ & $7.5-10$ & $10-12.5$ & $12.5-15$ & $15-20$ & $>20$ \\
\hline Spain-Germ. & 45.36 & 37.20 & 34.29 & 28.12 & 25.95 & 22.53 & 20.88 \\
Neth.-Germ. & 9.50 & 7.90 & 6.60 & 5.60 & 5.10 & 4.70 & 4.35 \\
\hline
\end{tabular}

The costs for replenishment orders were $18.04 \mathrm{Dfl} / 100 \mathrm{~kg}$ (including 3.00 holding and handling costs). The lower bound on the cost rate of transportation via the distribution centre equals $22.39(4.35+18.04) \mathrm{Dfl} / 100 \mathrm{~kg}$. In Figure 4.1 both the transportation tariff $T_{f c}^{r}(j)$ and the lower bound $\left(t_{f w}+t_{w c}^{r}\right) j$ are plotted, illustrating how the upper bound 
given in (2) can be determined. The flat parts in the tariff $T_{f c}^{r}(j)$ are caused by blanketing back (Ballou (1992)). For example, the cost $T_{f c}^{r}(21000)$ of transportating 21 tons from the factory in Spain to the customer in Germany is determined by $\min \left\{T_{f c}^{r}(20000), 20.88 *\right.$ $21000 / 100\}=T_{f c}^{r}(20000)=4506$. It can be verified that the upper bound given in $(2)$ equals $20125 \mathrm{~kg}$, i.e. $T_{f c}^{r}(20125)=4506=22.39 * 20125 / 100$.

Figure 4.1: Ilustration of determination upper bound on break quantity transportation costs (Dfl)

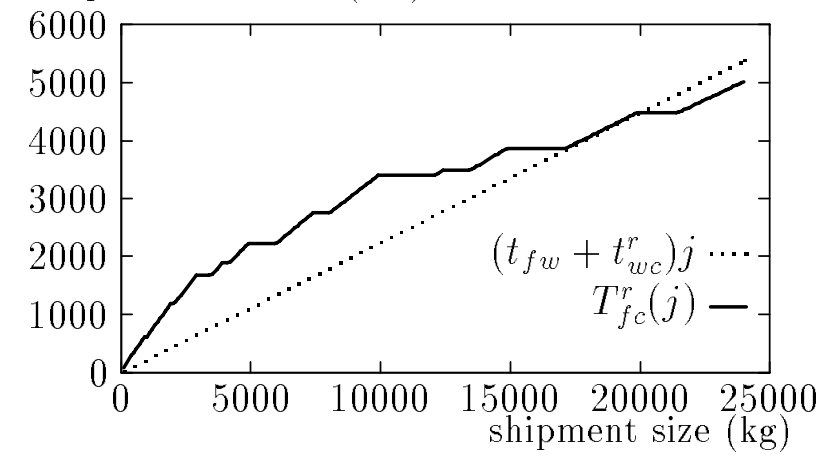

The 1994 demand of an arbitrary single customer, located in the region in Germany, is given in Table 4.2 .

Table 4.2: Demand (kg) of a customer during 1994

\begin{tabular}{c|cccccc}
\hline week & 1 & 8 & 11 & 14 & 22 & 27 \\
size $(\mathrm{kg})$ & 500 & 19800 & 4000 & 500 & 20 & 18750 \\
\hline week & 27 & 28 & 29 & 30 & 39 & \\
size $(\mathrm{kg})$ & 21875 & 10420 & 7920 & 21525 & 19540 & \\
\hline
\end{tabular}

For this particular customer we present the costs of the following different policies:

1. all direct, costs $\mathbf{3 1 0 7 2}$. With this policy all orders are delivered directly from the production plant in Spain.

2. all via $d c$, costs 29113. Here all the orders are delivered via the distribution centre. Observe that in week 27 two orders are consolidated.

3. upperbound, costs 28530. In this policy a break quantity rule is applied, with a break quantity equal to the upperbound $20125 \mathrm{~kg}$.

4. optimal break quantity, costs 28408. This policy uses a break quantity rule with the optimal break quantity (any size between 10420 and $18749 \mathrm{~kg}$ ), obtained by evaluating the total costs for all possible break quantities. 
The cost reduction obtained by using a break quantity rule with $q=20125$ instead of a policy where all orders are delivered via the distribution centre was $2 \%$. The cost difference between using the upper bound (2) instead of the optimal break quantity was only $0.4 \%$. With these observations we conclude our discussion of the case study.

\section{Conclusions}

In this chapter we discussed a tactical optimisation problem that arises when orders can be delivered from any stockpoint in the distribution system. A simple rule to allocate orders to locations is a break quantity rule, which routes large orders to higher-stage stockpoints (central warehouses, factories) and small orders to end-stockpoints (warehouses). A socalled break quantity determines whether an order is small or large.

The implications of a break quantity rule for the marketing process and the transportation, handling and inventory costs were described in Section 2, and in Section 3 a quantitative analysis of the impact on the transportation and inventory costs was presented. Summarising, the analysis for the transportation costs consists of comparing transportation tariffs for different break quantities, while the analysis for the inventory costs mainly focusses on the determination of the demand parameters, as a function of the break quantity, from which the average costs can be determined. The aggregate effect on both the transportation and the inventory costs should determine whether it is worthwhile to implement a break quantity rule, and if so, how large the break quantity should be. Finally, in Section 4 a case study was presented, illustrating an additional complexity that may arise in practice.

It is difficult to say if a break quantity rule will lead to a better performance of a distribution system, without looking closely at the marketing process and the transportation/inventory costs. However, in general it seems worthwhile to consider the implementation of a break quantity rule in distribution systems where demand is erratic (i.e. occasional very large demand transactions interspersed among a majority of small transactions) and the sizes of these large orders approach the full truck load size. For this situation, the reduction of the inventory and transportation costs will be significant if a break quantity rule is applied.

\section{References}

Adelson, R.M. (1996): Compound Poisson distributions, in: Operations Research Quarterly 17, 73-75. 
Ballou, R.H. (1992): Business Logistics Management, 3rd edition. (Prentice Hall) Englewood Cliffs, NJ.

Dekker, R./Frenk, J.B.G./Kleijn, M.J./Kok, A.G. de (1997): On the newsboy model with a cutoff transaction size. Technical Report 9736/A, Econometric Institute, Erasmus University Rotterdam, The Netherlands.

Dekker, R./Kleijn, M.J./Kok, A.G. de (1997): The break quantity rule's effect on inventory costs in a 1 -warehouse, $N$-retailers distribution system, to appear in: International Journal of Production Economics.

Eppen, G.D. (1979): Effect of centralization on expected costs in multi-location newsboy problem, in: Management Science 25, 498-501.

Fleischmann, B. (1993): Designing distribution systems with transport economies of scale, in: European Journal of Operational Research 70, 31-42.

Fleischmann, B. (1997): Design of freight traffic networks, in: Advances in Distribution Logistics, P. Stähly et al (editors).

Hollier, R.H./Mak, K.L./Lam, C.L. (1995a): Continuous review $(s, S)$ policies for inventory systems incorporating a cutoff transaction size, in: International Journal of Production Research 33, 2855-2865.

Hollier, R.H./Mak, K.L./Lam, C.L. (1995b): An inventory model for items with demands satisfied from stock or by special deliveries, in: International Journal of Production Economics 42, 229-236.

Kasturi Rangan, V./Jaikumar, R. (1991): Integrating distribution strategy and tactics: a model and an application, in: Management Science 37, 1377-1389.

Kok, A.G. de/Janssen, F.B.S.L.P. (1996): Demand management in multi-stage distribution chain. Technical Report 9639, Center for Economic Research, Tilburg University, The Netherlands.

Mak, K.L./Lai, K.K. (1995a): The determination of optimal partial fill policy for an inventory system with lumpy demand items, in: Applied Mathematical Modelling 19, $724-737$.

Mak, K.L./Lai, K.K. (1995b): Optimal $(s, S)$ policy for an inventory system when the demand can be partially satisfied, in: International Journal of Systems Science 26, $213-231$.

Nass, R./Dekker, R./Sonderen-Huisman, W. van (1997): Distribution optimization by means of break quantities: A case study, to appear in: Journal of the Operational Research Society. 
Silver, E.A. (1970): Some ideas related to the inventory control of items having erratic demand patterns, in: Journal of the Canadian Operations Research Society 8, 87-100.

Tijms, H.C. (1984): Stochastic Models: An Algorithmic Approach. (Wiley) New York. Tüshaus, U./Wahl, C. (1997): Inventory positioning in a two-stage distribution system with servicel level constraints, in: Advances in Distribution Logistics, P. Stähly et al (editors). 